\title{
Advanced Cardiac Life Support: 2016 Singapore Guidelines
}

\author{
Chi Keong Ching ${ }^{1}$, MBBS, MRCP, Siew Hon Benjamin Leong ${ }^{2}$, MBBS, MRCSEd, Siang Jin Terrance Chua ${ }^{1}$, MMed, MRCP, \\ Swee Han $\underline{\text { Lim}}^{3}$, FRCS, FRCP, Kenneth $\underline{\text { Heng }}^{4}$, MBBS, FRCS, Sohil Pothiawala ${ }^{3}$, FAMS, MRCSEd, \\ Venkataraman Anantharaman ${ }^{3}$, MBBS, FRCPEd; National Resuscitation Council Singapore
}

\begin{abstract}
The main areas of emphasis in the Advanced Cardiac Life Support (ACLS) guidelines are: early recognition of cardiac arrest and call for help; good-quality chest compressions; early defibrillation when applicable; early administration of drugs; appropriate airway management ensuring normoventilation; and delivery of appropriate post-resuscitation care to enhance survival. Of note, it is important to monitor the quality of the various care procedures. The resuscitation team needs to reduce unnecessary interruptions to chest compressions in order to maintain adequate coronary perfusion pressure during the ACLS drill. In addition, the team needs to continually look out for reversible causes of the cardiac arrest.
\end{abstract}

Keywords: advanced cardiac life support, cardiac arrest, cardiac drugs, cardiopulmonary resuscitation, reversible causes

\section{INTRODUCTION}

Advanced Cardiac Life Support (ACLS), the fourth link in the 'chain of survival' concept for sudden cardiac arrest, is highly dependent on the optimal conduct of the earlier three links, i.e. early access, early cardiopulmonary resuscitation (CPR) and early defibrillation. Arrhythmia management is a cornerstone of ACLS guidelines. Post-resuscitation interventions (i.e. measures carried out after return of spontaneous circulation [ROSC]) to increase the chance of being discharged alive from hospital have assumed increasing prominence. The 2016 ACLS guidelines address the following aspects of the vital fourth link in the chain of survival: (a) airway management; (b) vascular access and drug therapy; (c) identifying and treating reversible causes; (d) post-resuscitation care; (e) management of potentially unstable dysrhythmias; and ( $f$ ) organ donation. This follows on the earlier Singapore ACLS guidelines, which were published in 2011.(1)

\section{CARDIAC ARREST: EARLY RECOGNITION AND CALL FOR HELP}

Cardiac arrest may occur outside or within the hospital, and is termed out-of-hospital cardiac arrest (OHCA) or in-hospital cardiac arrest (IHCA), respectively.

\section{Out-of-hospital cardiac arrest}

In the out-of-hospital environment, the emphasis is on early recognition by the public and access to emergency prehospital care, prompt initiation of good-quality CPR by the bystander, public access defibrillation (PAD), early advanced care by emergency ambulance crew, and transport to hospital emergency departments (ED) that are capable of delivering resuscitation and post-resuscitation care.

\section{Dispatcher-assisted CPR}

Dispatcher-assisted CPR (DACPR) has been implemented in Singapore since 2011. It involves the 995 call-taker guiding the caller in recognising cardiac arrest through the key features of unresponsiveness and absence of normal breathing, followed by telephone instructions to provide chest compressions. DACPR has contributed to an increase in bystander CPR rates in Singapore. ${ }^{(2)}$

\section{Public access defibrillation}

PAD is another key intervention to improve the time to defibrillation for shockable rhythms in OHCA, through increasing the availability of automated external defibrillators (AEDs) in public places. Patients with shockable rhythms who received bystander defibrillation had 5.8 times the odds of survival compared to those who did not. ${ }^{(3)}$

\section{Emergency ambulance response}

Prehospital emergency medical services should optimise the rapid dispatch of first-responders who are trained in Basic Cardiac Life Support (BCLS) and AED use, as well as paramedics trained in advanced life support protocols, to the scene within eight minutes. This can be achieved through strategic preplacement of ambulances and related vehicular assets, as well as stratified levels of responses for calls of different priority levels.

Survival in OHCA is critically dependent on prompt institution of the first three links of the chain of survival. Prehospital factors, including witnessed arrests, initial shockable rhythms, bystander defibrillation and emergency ambulance response times within eight minutes of collapse, have been 
found to be associated with improved survival outcomes. ${ }^{(4)}$ As is well known, survival in OHCA decreases at a rate of $7 \%-10 \%$ with each passing minute without CPR after cardiac arrest. ${ }^{(5)}$ The low survival currently seen in many communities reflects the challenges in delivering timely and high-quality prehospital resuscitation care. The current overall survival rate of OHCA in Singapore is 2.5\%-3.2\%, with witnessed ventricular fibrillation (VF) survival rates at $11 \%$. There remains a need to better understand and address these gaps in the community. ${ }^{(3,6)}$ In addition, ensuring the best standard of ACLS in the hospital comprises an integral part of the continuum of resuscitation care for the OHCA victim.

The majority of cardiac arrests managed in EDs are OHCA. Most of these patients are conveyed by emergency ambulances and a small number by private transport or private ambulances. Some patients may go into cardiac arrest only after arriving at the ED. Limited data exists on the outcomes of such arrests, although survival factors may include patient acuity, comorbidities, early recognition and rapid response.

\section{In-hospital cardiac arrest}

A significant number of patients sustain cardiac arrests in the hospital environment. Some inpatients may have had a period of deterioration and an unwitnessed arrest. ${ }^{(7-9)}$ There may be significant variations in the time of detection of these cardiac arrests in the hospital. It is important to have a system for early recognition of patients at risk for cardiac arrest, through the use of early warning systems and detection of unexpected cardiac arrests, as well as a system of rapid response. For all IHCA patients, it is important to ensure the presence of the following: (a) early detection of cardiac arrest; (b) a system of calling for help within all clinical care areas, including inpatient wards, ambulatory clinics, operating theatres, intensive care units, and public areas of the hospital; (c) staff who are immediately available to perform CPR and operate an AED or defibrillator in the AED mode, as well as staff trained in ACLS interventions, at all times; and (d) resuscitation equipment, including defibrillators, advanced airway devices and drugs, is to be brought to the patient in the shortest possible time; the listing and layout for this equipment should preferably be standardised within each institution.

\section{Medical emergency and rapid response teams}

Various institutions have introduced medical emergency teams, rapid response teams, cardiac arrest teams or Code Blue teams to address the need to manage cardiac arrests. ${ }^{(10-13)}$ Institutions should have the capability to mobilise these teams promptly, so as to provide better oversight and management of resuscitations. Furthermore, it is important to ensure that all staff with patient care rights already possess a strong foundation in good basic and advanced life support skills. All clinical staff of healthcare facilities should also be expected to be currently certified in BCLS and $A E D$, unless they are excused owing to health reasons. In addition, all acute hospitals should have currently certified BCLSand ACLS-trained personnel who are available 24 hours a day.

\section{CHEST COMPRESSIONS IN CPR}

High-quality chest compressions remain the foundation of ACLS. However, the clinical contexts in which ACLS is delivered differ in in-hospital and out-of-hospital situations, as additional manpower, expertise and equipment are usually available in the hospital. The subsequent paragraphs detail recommendations for good-quality CPR in ACLS.

\section{Compression depth}

The depth of chest compressions is consistent with that stated in the BCLS guidelines, viz. $4-6 \mathrm{~cm}$ rather than at least $5 \mathrm{~cm}$, based on research demonstrating the optimal compression depths for survival. ${ }^{(14,15)}$

\section{Compression rate}

An upper limit of 120 beats per minute (bpm) has been added to the previous compression rate of at least $100 \mathrm{bpm}$, following evidence that excessive compression rates are associated with poorer outcomes. ${ }^{(16)}$ Excessive compression rates are counterproductive, as there is inadequate time for full chest recoil and, hence, inadequate ventricular filling.

\section{Chest recoil}

Complete chest recoil remains crucial to the quality of chest compressions, as it is during the relaxation phase that the myocardium is perfused. 'Residual leaning' on the chest after each compression by the rescuer impairs the coronary perfusion pressures and cardiac index, which are crucial for myocardial and systemic perfusion, respectively, ${ }^{(17)}$ during CPR. ACLS crew performing manual CPR must pay special attention to ensure complete chest recoil between compressions.

\section{Minimising interruptions}

Interruptions to CPR for ventilation, breathing and pulse check (if performed) or defibrillation should be less than ten seconds, in keeping with BCLS guidelines. Interruptions to CPR before and after defibrillation are collectively referred to as peri-shock pauses. Shorter peri-shock pauses are associated with improved survival. ${ }^{(18)}$ Interruptions to facilitate the insertion of advanced airways should also keep to this target.

\section{Real-time CPR monitoring and feedback}

CPR feedback systems and devices may improve the quality of CPR, although such devices or systems have not been found to improve survival outcomes. If utilised, real-time CPR monitoring and feedback should be part of a comprehensive response and quality framework for resuscitation.

\section{Automated mechanical CPR devices}

Automated mechanical CPR devices have been in use in prehospital and ED settings for many years. Two large studies ${ }^{(19,20)}$ on out-of-hospital use of mechanical CPR devices did not show any significant difference in outcomes between high-quality manual compressions and mechanical CPR devices on intentionto-treat analysis. However, in certain clinical situations, the 
provision of manual CPR may be impossible or potentially dangerous for the rescuer. These situations include performing resuscitation in moving ambulances, during patient transfers or in cardiac labs. Mechanical CPR also appears to have some theoretical advantages: it frees one pair of hands to assist in other areas of resuscitation; it can be started at home; it does not interrupt chest compressions for delivery of ventilation and defibrillatory shocks; and it can be continued while the patient is being moved (in the elevator and down steps), remaining stable in a moving ambulance and when the patient is being transferred to $\mathrm{ED}$ care. Thus, consideration should be given to the use of mechanical CPR devices during in-hospital resuscitation.

\section{VENTILATIONS IN CPR}

In contrast to BCLS, ACLS has the benefit of additional manpower, expertise and equipment. In the ACLS setting, there is no reason to omit ventilations in the provision of CPR. However, the question that arises is whether there is a need to synchronise ventilations with compressions during ACLS. A recent study published shortly after the 2015 International Liaison Committee on Resuscitation recommendations found no difference between continuous chest compressions with ten breaths delivered every minute and standard CPR with 30 compressions followed by two breaths prior to advanced airway management. ${ }^{(21)}$ On the basis of this new evidence, prior to advanced airway management in ACLS, the recommended rate of ventilation is 10 breaths/minute, or one breath every ten compressions when the compression rate is $100-120$ /minute. Standard CPR at 30:2 is equally acceptable. After an advanced airway is inserted, there is no further need to interrupt the compressions, and uninterrupted compressions at 100-120/minute with continuous ventilations at 10 breaths/minute should follow.

Care must be taken during the delivery of positive pressure ventilations. Unnecessarily high tidal volumes and ventilation rates are unhelpful, as cardiac output in CPR is only about $30 \%$ of normal, and pulmonary perfusion will thus be mismatched with excessive ventilation. Excessive bag-mask ventilation (BMV) leads to increased intrathoracic pressures that further decrease venous return and cardiac output. Gastric insufflation, regurgitation and aspiration, as well as diaphragmatic splinting that further impedes effective ventilation, can also occur. The recommended tidal volume is $400-600 \mathrm{~mL}$. If BMV is used, it is recommended that the user should compress the bag by about one-third, ${ }^{(22)}$ sufficient to produce a chest rise over one second.

\section{Rhythm check cycles}

Once a defibrillator or an AED is available, rhythm checks should be performed immediately and at every 1-2 minutes thereafter. The rhythm may be obtained using either electrocardiography (ECG) monitoring leads or self-adhesive defibrillator pads. Once the pads or ECG leads have been initially applied, CPR is interrupted for rhythm analysis. This is done manually by reading the rhythm on the ECG monitor of the defibrillator, or automatically by the AED or defibrillator in AED mode. Interruption to CPR during manual analysis should require no more than ten seconds. The duration of automated analyses by AEDs will vary depending on the model.

During the 1-2 minutes of CPR in-between rhythm analyses, there should be no interruptions to chest compressions. A common misconception is that the rescuer should pause chest compressions to analyse the rhythm if the rhythm changes during CPR. This practice should be stopped, because reacting to rhythm changes during CPR will only increase the number of interruptions to CPR. The team should only perform a rhythm check every 1-2 minutes, with good-quality CPR maintained throughout.

\section{Drugs in ACLS}

Drugs that may be used in refractory VF/polymorphic ventricular tachycardia (VT) include amiodarone and lignocaine in the doses described later in this article. Successful conversion with ROSC should be followed by an infusion of either amiodarone or lignocaine in the recommended doses.

\section{Nonshockable rhythms: pulseless electrical activity/ asystole}

In the absence of a shockable rhythm/asystole, other rhythms may be seen on the ECG monitor and a pulse check lasting no more than ten seconds may be performed. If no pulse can be reliably felt, then the rhythm is a form of pulseless electrical activity (PEA). CPR should be restarted immediately for 1-2 minutes before the next analysis.

If the rhythm monitor shows a flat line, perform the following checks to identify fine VF (do not take more than ten seconds to complete the checks): (a) check the connections to ensure that the leads are attached properly; (b) ensure that the cardiac monitor is not on paddle mode; (c) select different leads on the rhythm monitor to look for the presence of any fine VF along the different axes; and (d) ensure the gain/amplitude of the ECG tracing is normal. If the ECG rhythm remains a flat line despite these checks, the patient is in asystole. Commence CPR immediately for 1-2 minutes before the next analysis.

\section{Oxygen therapy in cardiac arrest}

The basic objective of ventilation in ACLS is to ensure oxygenation of tissues. Although there have been no studies directly comparing fractions of inspired oxygen delivered during resuscitation with survival, indirect studies have found that patients with higher $\mathrm{PaO}_{2}$ levels during resuscitation were more likely to achieve ROSC. ${ }^{(23)}$ From the currently available literature, it is recommended that $100 \%$ oxygen or the highest available oxygen concentrations be used during cardiac arrest resuscitation.

After ROSC, hyperoxaemia should be avoided during postcardiac arrest care. Several studies have found poorer neurological and survival outcomes with hyperoxaemia following ROSC.(24) After ROSC, oxygen therapy should be titrated to normoxia $\left(\mathrm{SpO}_{2} 94 \%-98 \%\right)$ as soon as possible.

\section{Airway control and management}

An open airway is crucial for the delivery of oxygen to the lungs and tissues. Access to the airway should be ensured within a 
few minutes of the start of resuscitation. All healthcare workers should be familiar with the skills of basic airway techniques. Basic airway opening techniques and principles include the following:

- The head-tilt, chin-lift manoeuvre remains the basic initial airway opening method.

- The classical or modified jaw thrusts are alternative methods, particularly in suspected cervical spine injury (e.g. patient with a history of fall or diving before cardiac arrest).

- Once opened, the airway needs to be cleared of secretions or foreign material. A blunt-tipped stiff suction catheter is recommended over flexible suction catheters.

- $\quad$ Airway adjuncts help to maintain patency of the oral or nasal air passages: (a) oropharyngeal airways prevent the tongue from occluding the upper airway, and it may be used in unresponsive patients with no cough or gag reflex, during BMV; (b) nasopharyngeal airways may be used if a clenched jaw prevents insertion of the oral airway; it has been known to cause bleeding in up to $30 \%$ of cases $^{(25)}$ and should be used with caution in the presence of craniofacial injury.

\section{Placement of advanced airways}

The aim of advanced airway management in ACLS is to ensure a secure passage for oxygen delivery and ventilation. The placement of endotracheal tubes (ETTs) or supraglottic airways is discussed below.

\section{Endotracheal tube}

Endotracheal intubation remains a core skill in advanced airway management. The benefits of ETT placement include allowing a definitive patent airway, suction of secretions, reliable oxygen delivery and protection from aspiration of gastric contents. It is indicated when BMV is ineffective or if the patient is in coma or cardiac arrest (absence of gag reflex).

\section{Cricoid pressure}

Cricoid pressure may offer some protection from aspiration and gastric insufflation. However, it may also impede ventilation and interfere with intubation. The routine use of cricoid pressure prior to intubation - a practice prior to the 2010 guidelines revision - is no longer recommended. ${ }^{(26)}$

\section{Confirmation of tube placement}

Following placement of an ETT, confirmation of placement is paramount, as missed oesophageal intubations are more harmful than no intubation. The expected standards for confirming correct placement clinically include: bilateral chest expansion; misting and demisting of ETT during BMV; and five-point auscultation, beginning from the epigastrium.

In addition to clinical assessment, continuous waveform capnography is recommended as a reliable method of confirming and monitoring correct placement of an ETT. If continuous waveform capnometry is not available, nonwaveform carbon dioxide detectors, oesophageal detector devices or ultrasonography used by an experienced operator are reasonable alternatives. Chest X-ray (CXR) should also be performed to assess the depth of ETT after a patient has achieved ROSC (of note, CXR cannot differentiate between an ETT in the oesophagus and one in the trachea, as both are midline structures).

\section{Complications}

Endotracheal intubation performed by an unskilled provider can result in the following: (a) trauma to the oropharynx with bleeding; (b) prolonged interruptions to compressions/ventilations; (c) prolonged hypoxaemia with resulting cerebral hypoxia; (d) failure to diagnose misplacement/displacement of tube, as recognised in $6 \%-25 \%$ of instances. ${ }^{(27-31)}$ Therefore, there is a need for regular training and retraining in these procedures to ensure currency of skills. Institutions must determine the frequency of such training and maintenance of competence standards for physicians who are expected to perform advanced airway placements. Vital signs monitoring during intubation for cardiac arrest situations is of limited value, as peripheral circulation is impaired and $\mathrm{SpO}_{2}$ measurement is ineffective or inaccurate. Following achievement of ROSC and adequate blood pressures, and in non-cardiac arrest situations such as elective or rapid sequence intubation, $\mathrm{SpO}_{2}$ monitoring is paramount.

\section{Supraglottic airways}

Supraglottic airways (SGAs) such as laryngeal mask airways, oesophageal tracheal airways (e.g. Combitube) or laryngeal tubes (e.g. King LT) are alternative advanced airway devices that may be used by prehospital care providers or by physicians if direct airway control is desired for short periods. Their main advantage is that direct visualisation of the glottis is not required, and training and skills maintenance are easier. If already inserted by emergency medical services for OHCA, or if cardiac arrest occurs while the patient has an SGA in situ such as during anaesthesia, the SGA may continue to be used for resuscitation, until equipment and expertise for endotracheal intubation are available.

Current evidence on the use of advanced airway devices (including both ETTs and SGAs) in the management of cardiac arrests has not proven that they improve outcomes over BMV. However, the majority of those studies were performed in out-ofhospital environments, predominantly by emergency prehospital personnel, and the evidence for advanced airways by trained and experienced physicians in hospitals is lacking. Airway interventions, when attempted, should not delay or interrupt chest compressions or attempts at defibrillation. If unsuccessful, BMV with basic airway management should be continued as a means of providing oxygenation for the patient.

\section{VASCULAR ACCESS AND DRUG THERAPY}

The use of drugs in cardiac arrest patients serves as an adjunct to the earlier components of care, viz. airway control and ventilation management, ensuring good-quality chest compressions with minimal interruptions and prompt arrhythmia management. The special features concerning the use of pharmacological agents during cardiac arrest management are elaborated below. 


\section{Routes of drug delivery}

- $\quad$ Peripheral large-calibre veins, especially the antecubital and external jugular veins, are the commonest routes to be used.

- Intraosseous cannulation can allow drug delivery to noncollapsible venous plexuses in the bone marrow. This may be rapidly inserted as an alternative route of access if intravenous (IV) access is not easily available. Rates of drug delivery are expected to be similar as for the IV route.

- Central venous lines, either through the subclavian or internal jugular veins, shorten medication access to the central circulation. Central lines, however, cannot be used for rapid infusion of fluids. The insertion of a central line is also likely to result in interruptions to chest compressions. Be aware of complications of central line insertion such as pneumothorax, and bleeding into the pleural space.

- $\quad$ ETT administration of drugs is no longer recommended because drug levels achieved are suboptimal and doses required to achieve blood levels that are similar to the IV route may be about 3-10 times as much. ${ }^{(32,33)}$

- Implanted vascular access devices, such as peripherally inserted central catheters or Port-a-caths, may be used with appropriate aseptic technique and care to avoid air emboli.

\section{Prolonged circulation time during CPR}

Circulation time during CPR is prolonged. Drugs will take 30-60 seconds of good-quality CPR to reach the central circulation and peripheral vasculature to exert their effects. Each drug dose should be flushed with a 20-mL bolus of normal saline. The administration of drugs should not interrupt CPR or delay the delivery of shocks, bearing in mind that the effect of the drug may not yet be evident until after the next cycle of CPR, owing to the time it takes to reach the central circulation.

\section{Common drugs used in resuscitation}

- $\quad$ Adrenaline is indicated for asystole, PEA and refractory VF. It should be administered in a 1.0-mg dose diluted to $10 \mathrm{~mL}$ $(1: 10,000)$ for bolus administration every $3-5$ minutes.

- Amiodarone is given intravenously at a dose of 300-mg bolus in refractory or recurrent VF/pulseless (i.e. VF or pulseless VT that persists or recurs after one or more shocks). This may be repeated at a dose of $150 \mathrm{mg}$, if necessary.

- Lignocaine is an alternative to amiodarone for patients in cardiac arrest due to refractory or recurrent VF/pulseless VT. ${ }^{(34)}$ It is usually given at a dose of $1-1.5 \mathrm{mg} / \mathrm{kg}$ body weight bolus.

Sodium bicarbonate $\left(\mathrm{NaHCO}_{3}\right)$ may be given for specific indications such as hyperkalaemia and tricyclic antidepressant overdose. It is usually given quickly as $1-1.5 \mathrm{~mL} / \mathrm{kg}$ body weight (i.e. $50-100 \mathrm{~mL}$ ) of IV $8.4 \% \mathrm{NaHCO}_{3}$. It is no longer recommended for routine administration in resuscitation from prolonged cardiac arrest.

\section{IDENTIFYING AND TREATING REVERSIBLE CAUSES}

Reversible causes of cardiac arrest, traditionally called the five $\mathrm{Hs}$ and five Ts (Box 1), should be evaluated for and treated if possible. The five Hs and five Ts are applicable to all cardiac arrest patients regardless of presenting rhythm and should be actively sought. Once reversible factors are identified, immediate action is required to correct these so as to provide the best conditions for a successful resuscitation. The specific clinical situation may identify the cause of cardiac arrest and offer prompt treatment, e.g. hyperkalaemia and hydrogen ion acidosis could be causes of cardiac arrest in patients on renal replacement therapy.

\begin{tabular}{|ll|}
\hline Box 1. The reversible causes of cardiac arrest: \\
Five Hs & Five Ts \\
- Hypoxia & - Toxic ingestions \\
- Hypovolaemia & - Tamponade (cardiac) \\
- Hydrogen ion acidosis & - Tension pneumothorax \\
- Hyper-/hypokalaemia & - Thrombosis, coronary or pulmonary \\
- Hypothermia & - Trauma \\
\hline
\end{tabular}

Ultrasonography may be used as an adjunct by trained physicians to detect reversible causes of cardiac arrest, as long as interruption to chest compressions is minimised or avoided. The evidence for the use of ultrasonography in cardiac arrest is currently limited.

\section{ACLS UNIVERSAL CARDIAC ARREST ALGORITHM}

The traditional model of separate rhythm-based algorithms for cardiac arrest has been modified into a single universal algorithm (Fig. 1) based on the principles described above. In practice, the progression of cardiac arrest does not follow a linear sequence according to any fixed rhythm, rendering separate algorithms unrealistic.

The universal algorithm adopts a cyclical model of analysis and management, with interventions employed when indicated. The patient enters the cycle with the primary ABCDs of basic airway opening, checking of breathing and CPR with defibrillation, then continues with the 1-2-minute rhythm check cycles, and exits the cycle upon ROSC. The ACLS interventions are performed concurrently by team members during the 1-2-minute cycles between rhythm checks. Reversible causes should be considered, looked for and treated during the resuscitation.

\section{POST-RESUSCITATION CARE}

When a pulse is present during the two-minute rhythm check, the patient has achieved ROSC. A system of integrated postcardiac arrest care ${ }^{(35)}$ is important to ensure survival and good neurological outcomes. This would include: (a) identification and treatment of the cause of cardiac arrest (e.g. percutaneous coronary intervention); (b) airway and ventilation management with an aim to prevent hypoxia and hyperoxia; (c) haemodynamic management to prevent hypotension and maintain mean arterial pressure of at least $90 \mathrm{mmHg}$; (d) targeted temperature management for at least 24 hours with gradual rewarming subsequently; (e) glycaemic control to maintain blood sugar level at 6-10 mmol/L; (f) seizure management; and (g) neuroprognostication after 72 hours. These measures are described in further detail in the paper on post-ROSC management. 


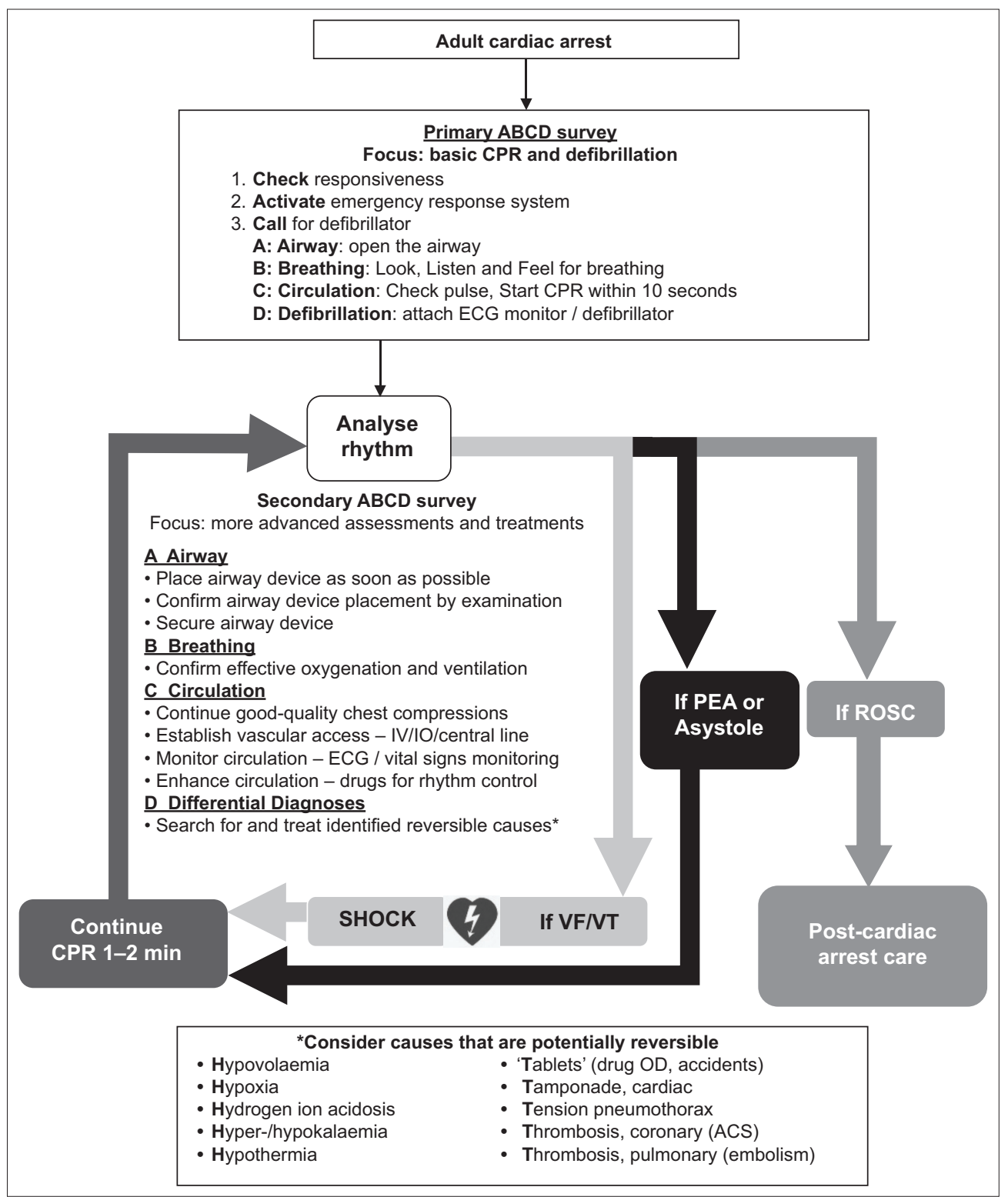

Fig. 1 Advance Cardiac Life Support algorithm.

\section{ORGAN DONATION}

Evidence ${ }^{(36-39)}$ suggests that functional outcomes of organs from patients deemed to be brain dead as a result of cardiac arrest are not significantly different from those of donors who are brain dead due to causes other than cardiac arrest. Many factors, including medical, social, cultural, legal and ethical issues, need to be considered when embarking on a programme of using viable organs from patients who are brain dead as a result of cardiac arrest. This is an area that needs careful assessment. It can potentially address community needs for scarce organs.

\section{PERI-ARREST ALGORITHM: POTENTIALLY UNSTABLE DYSRHYTHMIAS}

ACLS includes the management of potentially unstable dysrhythmias, such as tachydysrhythmia and bradydysrhythmia, in patients who are not in cardiac arrest. These dysrhythmias have the potential for deterioration, leading to impaired cardiac output, shock and cardiac arrest, and should thus be managed promptly. However, it must be remembered that other forms of shock, including hypovolaemic, haemorrhagic, distributive, obstructive and cardiogenic shock, may be present in the patient. Dysrhythmias constitute only one mechanism of cardiogenic shock. Rhythm control is part of the holistic management of the patient, which should include considerations of other mechanisms of shock and underlying conditions.

The general approach to dysrhythmia management has been standardised across the various algorithms. The management of each dysrhythmia is based on clinical stability. In general, hypotension, altered mental status, chest pain, breathlessness, signs of shock and heart failure are considered serious signs and symptoms that may be attributable to impaired cardiac output caused by the rhythm, thus suggesting haemodynamic instability. Patients should be managed with vital sign and ECG monitoring in clinical areas equipped for resuscitation. Supplemental oxygen should be given as necessary. A 12-lead ECG should be obtained as far as possible. If time does not allow, the ECG monitor may be utilised for rhythm assessment. 


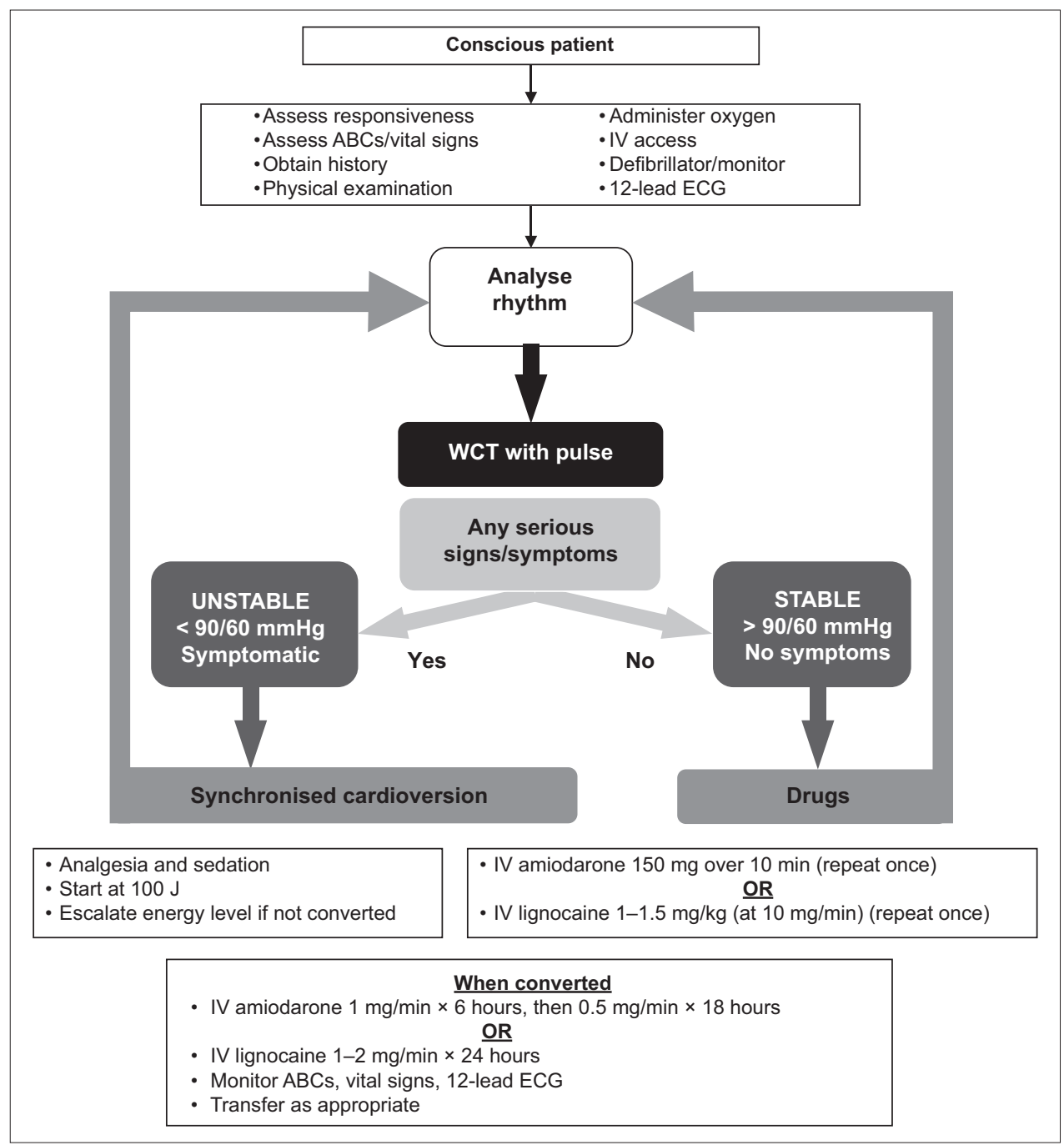

Fig. 2 Wide complex tachycardia (WCT) algorithm.

\section{WIDE COMPLEX TACHYCARDIAS}

The majority of wide complex tachycardias (WCTs) are due to VT. A small subset are due to supraventricular tachycardia (SVT) with aberrant conduction. Clinical (i.e. patient with a history of ischaemic heart disease) and ECG features specific to VT (i.e. atrioventricular dissociation capture beats and fusion beats) exist, but in the presence of clinical doubt, the patient should be managed as for VT. Fig. 2 shows the algorithm for WCT.

\section{Unstable patients}

Patients with WCTs who have serious signs and symptoms of instability should be treated rapidly with urgent synchronised cardioversion as follows: (a) explain the procedure to the patient; (b) give analgesia and sedation carefully in titrated doses (beware that drugs used in procedural sedation may have negative effects on haemodynamics); (c) energy levels should begin at $100 \mathrm{~J}$; evidence for different energy levels for monophasic or biphasic defibrillators is lacking; (d) ensure safety and clear as for defibrillation; (e) if the shock is unsuccessful, the energy level may be escalated and repeated $(150 / 200 \mathrm{~J} \rightarrow 360 \mathrm{~J})$ subject to the maximum energy level permitted by the individual defibrillator; and ( $\mathrm{f}$ ) once converted, an infusion of amiodarone at $1 \mathrm{mg} / \mathrm{min}$ for the first six hours followed by $0.5 \mathrm{mg} / \mathrm{min}$ for the next 18 hours, or lignocaine at $1-2 \mathrm{mg} / \mathrm{min}$ for 24 hours should be given.

\section{Stable patients}

If the patient is haemodynamically stable with no serious signs and symptoms, obtaining a 12-lead ECG first may provide additional useful information regarding the aetiology of the WCT.

- $\quad$ Patients with monomorphic VT may be managed with IV amiodarone $150 \mathrm{mg}$ diluted in $100 \mathrm{~mL}$ of D5W, given slowly over 10-15 minutes and repeated once if the first dose is unsuccessful.

- Upon conversion to sinus rhythm, continue with an infusion at $1 \mathrm{mg} / \mathrm{min}$ over six hours followed by $0.5 \mathrm{mg} / \mathrm{min}$ over the next 18 hours, up to $900 \mathrm{mg} /$ day.

- $\quad$ Alternatively, IV lignocaine at a dose of $1-1.5 \mathrm{mg} / \mathrm{kg}$ body weight may be administered at a rate of $10 \mathrm{mg} / \mathrm{min}$ and the dose repeated, if necessary, up to a maximum of $3 \mathrm{mg} / \mathrm{kg}$.

- Upon successful conversion to sinus rhythm, a maintenance infusion should be given at a rate of $1-2 \mathrm{mg} / \mathrm{min}$.

- It is reasonable to perform elective synchronised cardioversion if drug therapy fails. Transferring the patient 


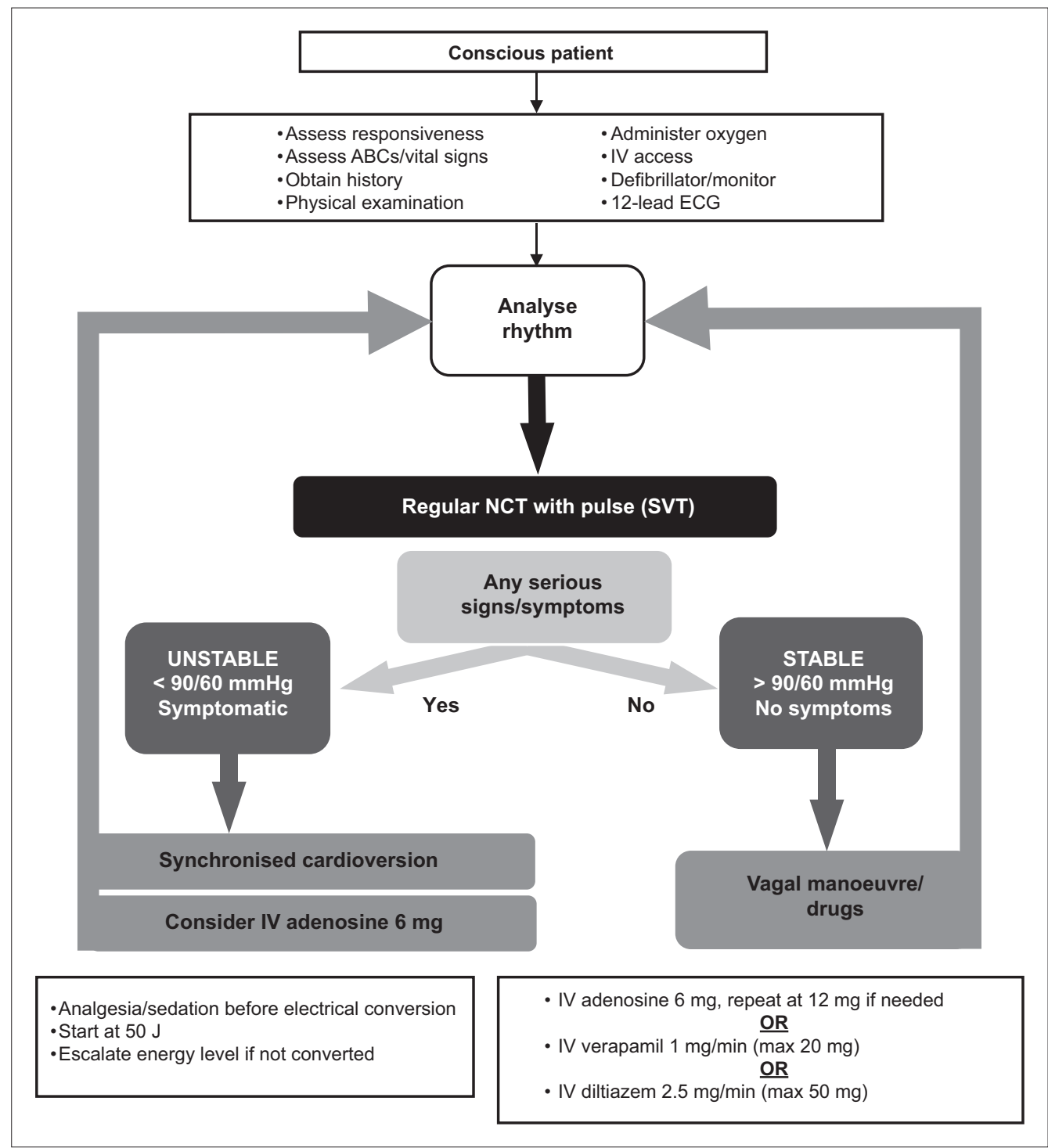

Fig. 3 Regular narrow complex tachycardia (NCT) algorithm.

to a monitored setting for this is preferred if the patient remains stable and time allows.

Polymorphic VT is a shockable rhythm and patients presenting with it are often haemodynamically unstable. Synchronised cardioversion may not be successful, as the computer in the defibrillator may be unable to find the R-wave to synchronise the shock to. Defibrillation may be necessary. If the cause of the polymorphic VT is a prolonged QT interval (torsades de pointes), intravenous magnesium sulphate 1-2 g over 15 minutes should be administered. In patients with polymorphic VT and normal QT interval, IV amiodarone $150 \mathrm{mg}$ over ten minutes may be given.

\section{NARROW COMPLEX TACHYCARDIAS}

The majority of regular narrow complex tachycardias (NCTs) are due to SVT and may also be due to atrioventricular nodal reentry or atrioventricular re-entry tachycardia. Patients presenting with SVT usually have a sudden onset of symptoms. Diagnosis is usually made after a 12-lead ECG is performed. Fig. 3 shows the NCT algorithm.

\section{Unstable patients}

Patients with regular NCTs who have serious signs and symptoms of instability should be treated with urgent synchronised cardioversion. The procedures for synchronised cardioversion are as follows: (a) explain the procedure to the patient; (b) give analgesia and sedation carefully in titrated doses (beware that drugs used in procedural sedation may have negative effects on haemodynamics); (c) energy levels should begin at 50 J; evidence for different energy levels for monophasic or biphasic defibrillators is lacking; (d) ensure safety and clear as for defibrillation; and (e) if the shock is unsuccessful, the energy level may be escalated and repeated.

One may consider the use of IV adenosine $6 \mathrm{mg}$ as a rapid bolus dose, followed by normal saline 20-mL flush for haemodynamically unstable patients with regular NCT. ${ }^{(40)}$

\section{Stable patients}

If vital signs are stable with no serious signs and symptoms, nonpharmacological cardioversion using vagal manoeuvres, such as carotid sinus massage or the modified Valsalva manoeuvre, may initially be attempted. 


\section{Valsalva manoeuvre}

The Valsalva manoeuvre achieves vagal stimulation by first causing an increase in intrathoracic pressures during patient straining through forced expiration against resistance, followed by sudden reduced intrathoracic pressures when the patient releases the strain. It is during the second phase, when intrathoracic pressures fall back to normal, that there is a sudden return of venous blood back into the central circulation. A surge in cardiac output follows, which triggers the vagal response. A recent postural modification to the traditional Valsalva manoeuvre has been published with some conversion success. ${ }^{(41)}$ The initial phase is performed with the patient sitting in a semirecumbent position, followed by laying the patient supine and passively elevating the patient's legs once the strain is released. This augments the venous return to the heart and accentuates the surge in cardiac output, thus triggering a more effective vagal response.

When performing the procedure: (a) explain the procedure to the patient; (b) position the patient in the trolley with the head of the trolley raised to a $45^{\circ}$ angle; (c) have the patient perform a forced expiration against resistance by blowing against the nozzle of a syringe or using a hand-held manometer for at least 15 seconds; (d) once the patient releases the strain, lower the head of the trolley to lay the patient flat and have an assistant raise the patient's legs to about $45^{\circ}$; and (e) monitor the rhythm for conversion.

\section{Carotid sinus massage}

Direct stimulation of the carotid sinuses stimulates a vagal response that may terminate the SVT. It should be avoided in older patients, patients with known cardiovascular diseases, and patients with history of transient ischaemic attack, stroke and the presence of carotid bruit, so as to avoid the risk of causing a stroke. When performing the procedure: (a) explain the procedure to the patient; (b) lay the patient flat or in a Trendelenburg position to distend the carotid sinus; (c) localise the carotid artery by feeling for the pulse in the groove between the trachea and sternocleidomastoid muscle; (d) trace the carotid artery upwards until close to the carotid bifurcation where the carotid sinus with its rich plexus of nerves lies; (e) apply firm pressure on this site by pressing the carotid artery against the transverse processes of the cervical vertebrae using the thumb or fingers in circular or linear motion for 10-15 seconds; (f) monitor the cardiac rhythm for conversion, and the patient for changes in conscious level; and (g) if unsuccessful, massage of the opposite carotid sinus may be attempted.

\section{Pharmacological cardioversion}

In the event of unsuccessful attempts with vagal manoeuvres, pharmacological agents may be employed to achieve rhythm control.

- IV adenosine, verapamil and diltiazem are all acceptable drugs of first choice for the conversion of stable patients with SVT.

- Adenosine for pharmacological cardioversion of SVT is given as a rapid IV bolus of $6 \mathrm{mg}$ via a proximal large calibre vein, followed immediately by a $20-\mathrm{mL}$ saline flush using a three-way connector. This may be repeated at $12 \mathrm{mg}$ if the initial bolus is unsuccessful. The patient must be warned of side effects of transient chest tightness, bronchospasm and hot facial flushes soon after drug administration.

- A simplified method of administration has been described, ${ }^{(42)}$ where the dose of adenosine is first mixed into the saline flush and the mixture administered as a single bolus. There is no difference in success rates, so this method may be considered an alternative for adenosine administration.

- IV verapamil, a calcium channel blocker, may also be used for the treatment of SVT. It is given as an infusion at a rate of $1 \mathrm{mg} / \mathrm{min}$ up to a maximum of $20 \mathrm{mg}$ with haemodynamic monitoring at two-minute intervals. ${ }^{(43)}$ The infusion is stopped once sinus rhythm is achieved. In patients with atrial fibrillation (AF) with a fast ventricular rate, it may be used as a rate control agent when infused at the rate of $1 \mathrm{mg}$ over three minutes up to a maximum of $20 \mathrm{mg}$, again with haemodynamic monitoring. It should be avoided in the presence of heart failure owing to a possible negative inotropic effect.

- $\quad$ IV diltiazem is a calcium channel blocker. For patients with SVT, it may be infused at a rate of $2.5 \mathrm{mg} / \mathrm{min}$ up to a maximum of $50 \mathrm{mg}$ with haemodynamic monitoring at twominute intervals. The infusion is stopped once conversion to sinus rhythm is achieved. ${ }^{(44)}$ In patients with AF with rapid ventricular response, it may be infused at the rate of $2.5 \mathrm{mg}$ over three minutes up to a maximum dose of $50 \mathrm{mg}$, again with haemodynamic monitoring, to achieve controlled ventricular response. It should be avoided in the presence of heart failure owing to a possible negative inotropic effect.

- $\quad$ ECG and vital signs monitoring should be continued during the process and for at least two hours thereafter, for early detection of recurrence.

\section{ATRIAL FIBRILLATION}

The algorithm for AF is shown in Fig. 4.

\section{Unstable patients}

Patients presenting with AF with rapid ventricular response may develop haemodynamic or clinical instability. Bear in mind that patients with fast ventricular rates in AF may have other causes of instability such as sepsis or dehydration. AF patients with serious signs and symptoms should be managed in a monitored area.

- If the rhythm is assessed to be the primary cause of instability, synchronised cardioversion with procedural sedation would be required for urgent rhythm control.

- $\quad$ The shock energy should begin at $100 \mathrm{~J}$ and escalated if necessary. Lower shock energies are unlikely to be effective for AF.

- The coagulation status for the patient should be checked, and IV heparin given before cardioversion.

- $\quad$ The procedure for performing cardioversion is no different from that for unstable WCT or NCT. 


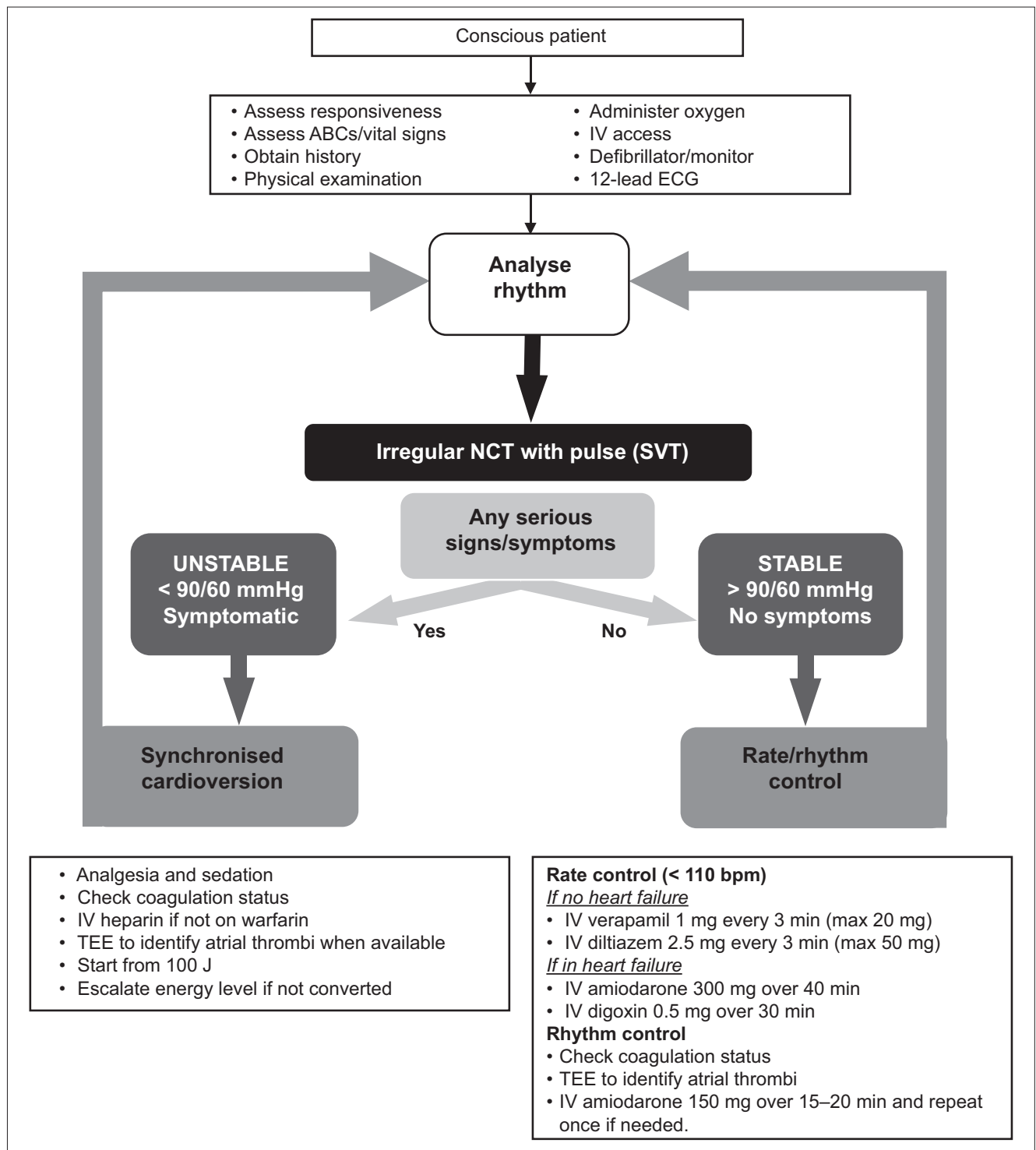

Fig. 4 Irregular narrow complex tachycardia (NCT) algorithm.

\section{Stable patients}

In stable patients, the urgency for rhythm control is no longer present. The priority then becomes rate control.

- $\quad$ For rate control of AF, calcium channel blockers, such as verapamil or diltiazem, given as slow infusions may be considered in the absence of heart failure. Short-acting beta-blockers are suitable alternatives, particularly for AF associated with thyrotoxicosis. For patients with heart failure, the current recommendation is infusion of amiodarone 300 g over a 40-minute period, with frequent monitoring of blood pressure owing to the blood pressurelowering effects of amiodarone. Care should be taken in patients with thyroid disease. IV digoxin $0.5 \mathrm{mg}$ infused over 30 minutes is an alternative, but will take effect only several hours later. ${ }^{(45)}$

- For rhythm control of AF (i.e. pharmacological cardioversion), IV amiodarone 150 mg over 20 minutes may be administered and repeated if there is failure of conversion with the initial dose. Vital signs monitoring will be required during the infusion. As patients for whom pharmacological cardioversion is being considered are typically haemodynamically stable, transoesophageal echocardiography and heparin administration should be performed first. Elective electrical cardioversion may also be considered for stable AF patients.

\section{BRADYARRHYTHMIAS}

Haemodynamically significant bradyarrhythmias usually present with a heart rate of below $60 \mathrm{bpm}$ and a blood pressure of less than $90 / 60 \mathrm{mmHg}$. Most patients who are symptomatic would tend to have heart rates that are even lower than 50 bpm. Asymptomatic and haemodynamically stable patients generally do not need further treatment for the bradycardia. However, patients with Mobitz Type II second degree or complete heart blocks should be monitored closely even if stable, as they are at risk for sudden deterioration. Unstable patients should be managed in a monitored area and given supplemental oxygen or ventilatory assistance as appropriate. A 12-lead ECG or rhythm strip should be obtained to analyse the rhythm. The algorithm for bradyarrhythmias is shown in Fig. 5. 


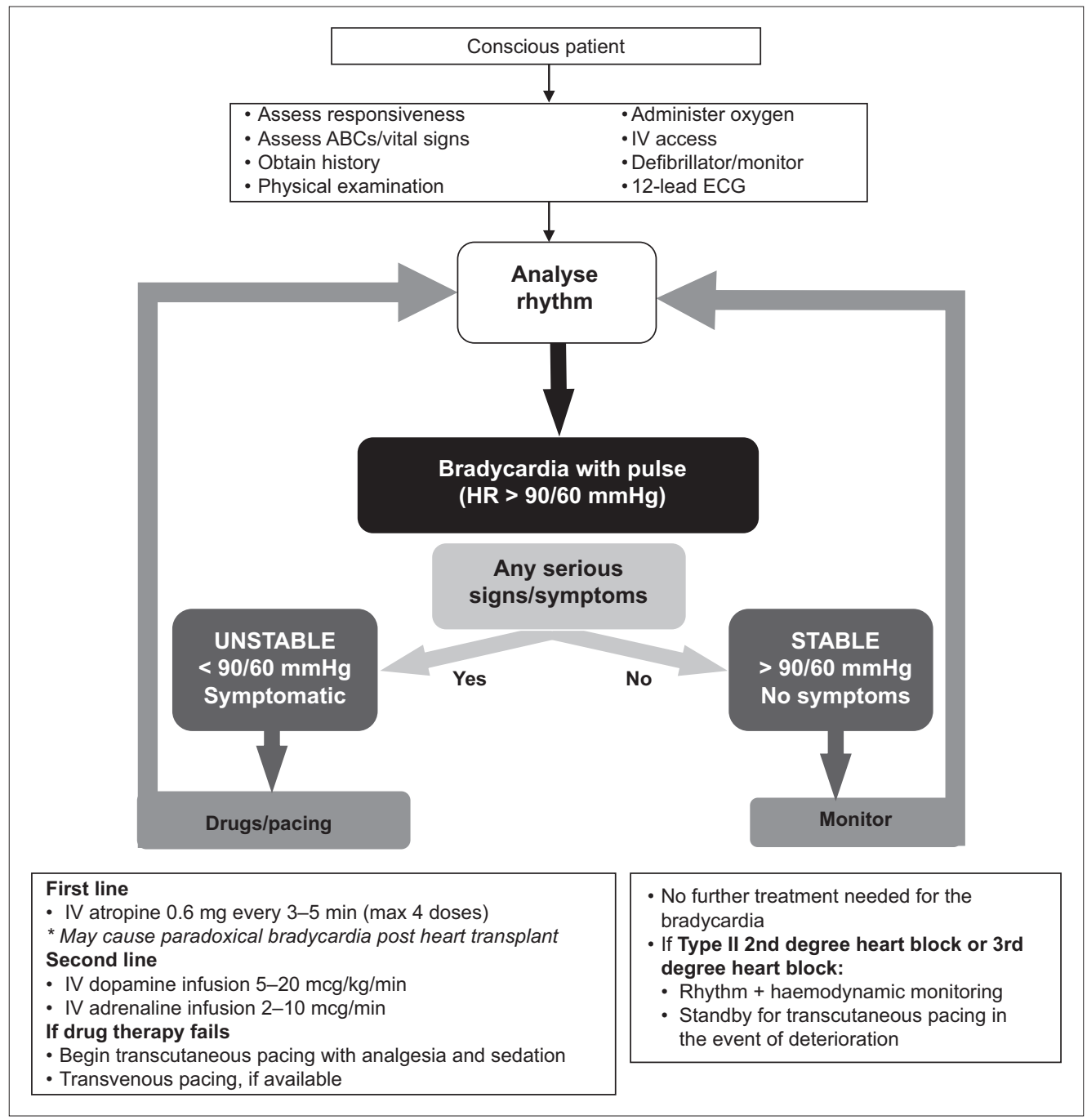

Fig. 5 Bradycardia algorithm.

The common drugs that may be used for bradyarrhythmias are atropine, dopamine infusion and adrenaline infusion.

- Atropine for bradycardia is given in repeated boluses of $0.6 \mathrm{mg}$ up to a maximum vagolytic dose of $2.4 \mathrm{mg}$.

- Dopamine infusion is started at a rate of $5-20 \mathrm{mcg} / \mathrm{kg} / \mathrm{min}$ and increased to a maximum of $20 \mathrm{mcg} / \mathrm{kg} / \mathrm{min}$, above which the likelihood of peripheral and splanchnic vasoconstriction may be significant and undesirable.

- Adrenaline infusions for bradycardia are initiated at a rate of $2-10 \mathrm{mcg} / \mathrm{min}$ and increased gradually till the target heart rate and/or blood pressure is achieved.

- Cardiac pacing would have to be considered for all patients with haemodynamically significant bradycardias. In an emergency situation, transcutaneous pacing is the preferred method until transvenous pacing can be organised. Transcutaneous pacing is painful in conscious patients, requiring analgesia and sedation.

\section{REFERENCES}

1. Anantharaman V, Gunasegaran K. Advanced Cardiac Life Support guidelines 2011. Singapore Med J 2011; 52:548-55; quiz 556

2. Harjanto S, Na MX, Hao Y, et al; PAROS study group. A beforeafter interventional trial of dispatcher assisted cardio-pulmonary resuscitation for out-of-hospital cardiac arrests in Singapore. Resuscitation 2016; 102:85-93.

3. Lai $\mathrm{H}$, Choong CV, Fook-Chong $\mathrm{S}$, et al; PAROS study group. Interventional strategies associated with improvements in survival for out-of-hospital cardiac arrests in Singapore over 10 years. Resuscitation 2015; 89:155-61.

4. Ong ME, Yan X, Lau G, et al. Out-of-hospital cardiac arrests occurring in primary health care facilities in Singapore. Resuscitation 2007; 74:38-43.

5. Larsen MP, Eisenberg MS, Cummins RO, Hallstrom AP. Predicting survival from out-of-hospital cardiac arrest: a graphic model. Ann Emerg Med 1993; 22:1652-8.

6. Ong ME, Shin SD, De Souza NN, et al; PAROS Clinical Research Network. Outcomes for out-of-hospital cardiac arrests across 7 countries in Asia: The Pan Asian Resuscitation Outcomes Study (PAROS). Resuscitation 2015; 96:100-8.

7. Cullinane M, Findlay G, Hargraves C, Lucas S. An Acute Problem? London: National Confidential Enquiry into Patient Outcome and Death. Available at: http://www.ncepod.org.uk/2005report/. Accessed March 31, 2017.

8. Hodgetts TJ, Kenward G, Vlackonikolis I, et al. Incidence, location and reasons for avoidable in-hospital cardiac arrest in a district general hospital. Resuscitation 2002; 54:115-23

9. Kause J, Smith G, Prytherch D, et al; Intensive Care Society (UK); Australian and New Zealand Intensive Care Society Clinical Trials Group. A comparison of antecedents to cardiac arrests, deaths and emergency intensive care admissions in Australia and New Zealand, and the United Kingdom--the ACADEMIA study. Resuscitation 2004; 62:275-82.

10. Baxter AD, Cardinal P, Hooper J, Patel R. Medical emergency teams at The Ottawa Hospital: the first two years. Can J Anaesth 2008; 55:223-31.

11. Benson L, Mitchell C, Link M, Carlson G, Fisher J. Using an advanced practice nursing model for a rapid response team. Jt Comm J Qual Patient Saf 2008; 34:743-7.

12. Buist MD, Moore GE, Bernard SA, et al. Effects of a medical emergency team on reduction of incidence of and mortality from unexpected cardiac arrests in 
hospital: preliminary study. BMJ 2002; 324:387-90.

13. Buist M, Harrison J, Abaloz E, Van Dyke S. Six year audit of cardiac arrests and medical emergency team calls in an Australian outer metropolitan teaching hospital. BMJ 2007; 335:1210-2.

14. Stiell IG, Brown SP, Nichol G, et al; Resuscitation Outcomes Consortium Investigators. What is the optimal chest compression depth during out-of-hospital cardiac arrest resuscitation of adult patients? Circulation 2014; 130:1962-70.

15. Hellevuo H, Sainio M, Nevalainen R, et al. Deeper chest compression - more complications for cardiac arrest patients? Resuscitation 2013; 84:760-5.

16. Abella BS, Sandbo N, Vassilatos P, et al. Chest compression rates during cardiopulmonary resuscitation are suboptimal: a prospective study during inhospital cardiac arrest. Circulation 2005; 111:428-34.

17. Niles DE, Sutton RM, Nadkarni VM, et al. Prevalence and hemodynamic effects of leaning during CPR. Resuscitation 2011; 82 Suppl 2:S23-6.

18. Cheskes S, Schmicker RH, Christenson J, et al; Resuscitation Outcomes Consortium (ROC) Investigators. Perishock pause: an independent predictor of survival from out-of-hospital shockable cardiac arrest. Circulation 2011; 124:58-66.

19. Wik L, Olsena J, Persse D, et al. Manual vs. integrated automatic load-distributing band CPR with equal survival after out of hospital cardiac arrest. The randomized CIRC trial. Resuscitation 2014; 85:741-8.

20. Rubertsson S, Lindgren E, Smekal D, et al. Mechanical chest compressions and simultaneous defibrillation vs conventional cardiopulmonary resuscitation in out-of-hospital cardiac arrest: The LINC Randomized Trial. JAMA 2014 311:53-6.

21. Nichol G, Leroux B, Wang $\mathrm{H}$, et al; ROC Investigators. Trial of continuous or interrupted chest compressions during CPR. N Engl J Med 2015; 373:2203-14.

22. Cho YC, Cho SW, Chung SP, et al. How can a single rescuer adequately delive tidal volume with a manual resuscitator? An improved device for delivering regular tidal volume. Emerg Med J 2011; 28:40-3.

23. Takei $Y$, Enami M, Yachida T, Ohta K, Inaba H. Tracheal intubation by paramedics under limited indication criteria may improve the short-term outcome of out-of-hospital cardiac arrests with noncardiac origin. J Anesth 2010; 24:716-25.

24. Kilgannon JH, Jones AE, Shapiro NI, et al; Emergency Medicine Shock Research Network (EMShockNet) Investigators. Association between arterial hyperoxia following resuscitation from cardiac arrest and in-hospital mortality. JAMA 2010; 303:2165-71.

25. Stoneham MD. The nasopharyngeal airway. Assessment of position by fibreoptic laryngoscopy. Anaesthesia 1993; 48:575-80.

26. Hartsilver EL, Vanner RG. Airway obstruction with cricoid pressure. Anaesthesia 2000; 55:208-11.

27. Jones JH, Murphy MP, Dickson RL, Somerville GG, Brizendine EJ. Emergency physician-verified out-of-hospital intubation: miss rates by paramedics. Acad Emerg Med 2004; 11:707-9.

28. Sayre MR, Sakles JC, Mistler AF, et al. Field trial of endotracheal intubation by basic EMTs. Ann Emerg Med 1998; 31:228-33.

29. Katz SH, Falk JL. Misplaced endotracheal tubes by paramedics in an urban emergency medical services system. Ann Emerg Med 2001; 37:32-7.

30. Jemmett ME, Kendal KM, Fourre MW, Burton JH. Unrecognized misplacemen of endotracheal tubes in a mixed urban to rural emergency medical services setting. Acad Emerg Med 2003; 10:961-5.

31. Silvestri S, Ralls GA, Krauss B, et al. The effectiveness of out-of-hospital use of continuous end-tidal carbon dioxide monitoring on the rate of unrecognized misplaced intubation within a regional emergency medical services system. Ann Emerg Med 2005; 45:497-503.

32. Schüttler J, Bartsch A, Ebeling BJ, et al. [Endobronchial administration of adrenaline in preclinical cardiopulmonary resuscitation]. Anasth Intensivther Notfallmed 1987; 22:63-8. German.

33. Hörnchen U, Schüttler J, Stoeckel H, Eichelkraut W, Hahn N. Endobronchial instillation of epinephrine during cardiopulmonary resuscitation. Crit Care Med 1987; 15:1037-9.

34. Kudenchuk PJ, Brown SP, Daya M, et al; Resuscitation Outcomes Consortium Investigators. Amiodarone, lidocaine, or placebo in out-of-hospital cardiac arrest. N Engl J Med 2016; 374:1711-22

35. Peberdy MA, Callaway CW, Neumar RW, et al; American Heart Association. Part 9: post-cardiac arrest care: 2010 American Heart Association Guidelines for Cardiopulmonary Resuscitation and Emergency Cardiovascular Care. Circulation 2010; 122(18 Suppl 3):S768-86

36. Adrie $\mathrm{C}$, Haouache $\mathrm{H}$, Saleh $\mathrm{M}$, et al. An underrecognized source of organ donors: patients with brain death after successfully resuscitated cardiac arrest. Intensive Care Med 2008; 34:132-7.

37. Ali AA, Lim E, Thanikachalam M, et al. Cardiac arrest in the organ donor does not negatively influence recipient survival after heart transplantation. Eur J Cardiothorac Surg 2007; 31:929-33.

38. Matsumoto CS, Kaufman SS, Girlanda R, et al. Utilization of donors who have suffered cardiopulmonary arrest and resuscitation in intestinal transplantation. Transplantation 2008; 86:941-6.

39. Mercatello A, Roy P, Ng-Sing K, et al. Organ transplants from out-of-hospital cardiac arrest patients. Transplant Proc 1988; 20:749-50

40. Gebril A. Is intravenous adenosine effective and safe in patients presenting with unstable paroxysmal supraventricular tachycardia? In: BestBets [online]. Available at: http://bestbets.org/bets/bet.php?id=181. Accessed August 4, 2016.

41. Appelboam A, Reuben A, Mann C, et al; REVERT trial collaborators. Postural modification to the standard Valsalva manoeuvre for emergency treatment of supraventricular tachycardias (REVERT): a randomised controlled trial. Lancet 2015; 386:1747-53.

42. Choi SC, Yoon SK, Kim GW, et al. A convenient method of adenosine administration for paroxysmal supraventricular tachycardia. J Korean Soc Emerg Med 2003; 14:224-7.

43. Lim SH, Anantharaman V, Teo WS, Chan YH. Slow infusion of calcium channel blockers compared with intravenous adenosine in the emergency treatment of supraventricular tachycardia. Resuscitation 2009; 80:523-8.

44. Lim SH, Anantharaman V, Teo WS. Slow-infusion of calcium channel blockers in the emergency management of supraventricular tachycardia. Resuscitation 2002; 52:167-74

45. Hofmann R, Steinwender C, Kammler J, Kypta A, Leisch F. Effects of a high dose intravenous bolus amiodarone in patients with atrial fibrillation and a rapid ventricular rate. Int J Cardiol 2006; 110:27-32 


\section{SINGAPORE MEDICAL COUNCIL CATEGORY 3B CME PROGRAMME} (Code SMJ 201707B)

Question 1. If an electrocardiography (ECG) monitor-cum-defibrillator is available when resuscitating an adult patient in cardiac arrest:

(a) Rhythm checks should be done every 3-5 minutes.

(b) The first shock must be delivered once the ECG machine is connected.

(c) Once a defibrillatory shock is delivered, the rhythm should first be checked before any further chest compressions are given.

(d) Once a defibrillatory shock is delivered, CPR should be resumed immediately for 1-2 minutes before the ECG rhythm is checked.

Question 2. The following statement(s) is/are true regarding drug use in patients with refractory ventricular fibrillation (VF):

(a) Amiodarone is superior to lignocaine and adrenaline for conversion of witnessed VF.

(b) The recommended dose of adrenaline is $1.0 \mathrm{mg}$ given as a $1.0 \mathrm{~mL}$ bolus.

(c) The recommended dose of amiodarone is $300 \mathrm{mg}$ as a first dose and $150 \mathrm{mg}$ every 3-5 minutes later, if $\mathrm{VF}$ persists.

(d) The recommended dose of lignocaine is $1-1.5 \mathrm{mg} / \mathrm{kg}$ body weight repeated $3-5$ minutes later, if $\mathrm{VF}$ persists.

Question 3. Regarding reversible causes of cardiac arrest:

(a) Hypoxia, hyperkalaemia and metabolic acidosis should be considered in all patients with pulseless electrical activity (PEA) only.

(b) Hypoxia, hyperkalaemia and hydrogen ion acidosis should be considered in all patients with refractory VF.

(c) Use of cardiac ultrasonography is mandatory in all patients in cardiac arrest so that reversible causes may be identified.

(d) Infusing intravenous $8.4 \%$ sodium bicarbonate in cardiac arrest patients prevents PEA that may result from acidosis.

Question 4. In patients with wide complex tachycardia:

(a) Intravenous adenosine $6.0 \mathrm{mg}$ should be initially administered to rule out supraventricular tachycardia with aberrant conduction.

(b) In a 66-year-old man with wide complex tachycardia, the initial management should be the same as that for ventricular tachycardia (VT).

(c) A patient with VT and a blood pressure of $85 / 50 \mathrm{mmHg}$ should be immediately defibrillated with unsynchronised shocks of $100 \mathrm{~J}$ of biphasic energy.

(d) Amiodarone and lignocaine are both drugs of choice for those with stable VT.

Question 5. In a patient presenting with narrow complex tachycardia:

(a) Either synchronised cardioversion at $50 \mathrm{~J}$ after sedation or intravenous adenosine $6 \mathrm{mg}$ may be used if the patient has a regular heart rate of 180 beats per minute and a blood pressure of $74 / 46 \mathrm{mmHg}$.

(b) In addition to intravenous adenosine, intravenous verapamil and diltiazem as slow infusions are acceptable first-line agents for chemical conversion of supraventricular tachycardia.

(c) Intravenous verapamil and intravenous diltiazem are not advised if the patient has an irregular rhythm at 180 beats per minute and heart failure.

(d) Carotid sinus massage for conversion of the patient's narrow complex tachycardia involves application of pressure at the midpoint of the common carotid artery at the anterior edge of the sternomastoid muscle.

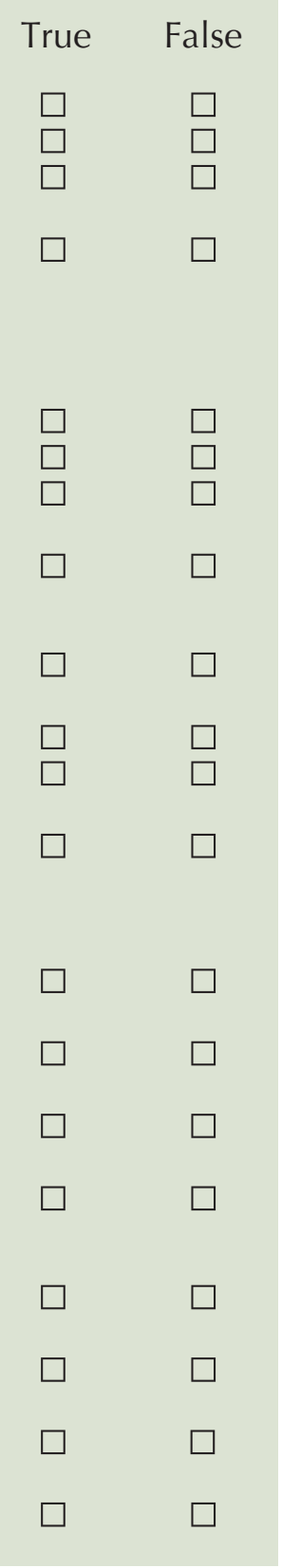

\section{Doctor's particulars:}

Name in full:

MCR no.:

Specialty:

Email:

\section{SUBMISSION INSTRUCTIONS:}

Visit the SMJ website: http://www.smj.org.sg/current-issue and select the appropriate quiz. You will be redirected to the SMA login page.

For SMA member: (1) Log in with your username and password (if you have not activated your membership account, please email membership@sma.org.sg). (2) Select your answers for each quiz and click 'Submit'.

For non-SMA member: (1) Create an SMJ-CME account, or log in with your SMJ-CME username and password (for returning users). (2) Make payment of SGD 21.40 (inclusive of $7 \%$ GST) via PayPal to access this month's quizzes. (3) Select your answers for each quiz and click 'Submit'.

\section{RESULTS:}

(1) Answers will be published online in the SMJ September 2017 issue. (2) The MCR numbers of successful candidates will be posted online at the SMJ website by 14 September 2017. (3) Passing mark is $60 \%$. No mark will be deducted for incorrect answers. (4) The SMJ editorial office will submit the list of successful candidates to the Singapore Medical Council. (5) One CME point is awarded for successful candidates.

Deadline for submission: (July 2017 SMJ 3B CME programme): 12 noon, 7 September 2017. 